\title{
Cambios en cobertura y regeneración arbórea en bosques siempreverdes en diferentes estados sucesionales en el sitio experimental de Llancahue, Cordillera de la Costa de Valdivia, Chile
}

\section{Changes in cover and tree regeneration in evergreen forests in different successional stages in the Llancahue experimental site, Coastal range of Valdivia, Chile}

\author{
Pablo J. Donoso ${ }^{1 *}$, Diego B. Ponce ${ }^{1}$, Joselyn B. Pinto ${ }^{1}$ \& Iván L. Triviño 2 \\ ${ }^{1}$ Instituto de Bosques y Sociedad, Universidad Austral de Chile, Valdivia, Chile. \\ ${ }^{2}$ Instituto de Ciencias Marinas y Limnológicas, Universidad Austral de Chile, Valdivia, Chile. \\ *pdonoso@uach.cl
}

\begin{abstract}
In the Llancahue experimental site $\left(39^{\circ} 50^{\prime} ; 300-400 \mathrm{~m}\right.$ ) we evaluated richness of tree and understory species in an oldgrowth forest and in two secondary forests. Having similar species' richness in these forests, the study supports our hypothesis that this is a result of successional development and natural and anthropogenic disturbances.
\end{abstract}

Los bosques nativos de altitudes bajas de las cordilleras y de la depresión intermedia del centro-sur de Chile han sufrido severos niveles de disminución o fragmentación durante el último siglo, especialmente debido a cambios de uso de la tierra o explotación forestal no sustentable (Lara et al. 2016). Esta pérdida ha afectado principalmente a bosques adultos, pero incluso los bosques secundarios se encuentran altamente fragmentados (Locher-Krause 2017). En consecuencia, es cada vez más difícil en esta región encontrar superficies continuas y contiguas de bosques adultos y secundarios en buen estado de conservación. En este sentido, las reservas de bosques nativos de altitudes bajas y en buen estado de conservación en el centro-sur de Chile adquieren un creciente valor, ya que son sitios únicos para conocer las características vegetacionales de estos bosques y para monitorear cambios derivados de la dinámica natural o perturbaciones exógenas en estos ecosistemas.

El estudio se desarrolló en el sitio experimental Llancahue $\left(39^{\circ} 49^{\prime} \mathrm{S}, 73^{\circ} 07^{\prime} \mathrm{O}\right)$, una reserva fiscal de 1270 ha de bosques adultos y secundarios en muy buen estado de conservación (Fig. 1) y que provee múltiples servicios ecosistémicos (Donoso et al. 2014). En este sitio se instalaron nueve unidades muestrales cuadradas de 1 ha cada una, con tres unidades representando a cada uno de tres tipos de bosques (Tabla 1), todos ubicados entre los 300 y 400 m s.n.m., en suelos de la serie Los Ulmos (CIREN $1999)$, y sobre pendientes suaves $(<20 \%)$. Los tres bosques corresponden al Tipo Forestal Siempreverde descrito por Donoso (2015) o al Bosque Laurifolio Templado Interior de Nothofagus dombeyi y Eucryphia cordifolia descrito por Luebert \& Pliscoff (2006). Dos de los bosques muestreados corresponden a bosques nativos secundarios en distintos estados sucesionales (temprano e intermedio) y uno a un bosque adulto (BA) en un avanzado estado sucesional. El bosque en un estado sucesional más temprano corresponde a un bosque secundario mixto siempreverde (BSMS) que fue originado por madereo y presumiblemente fuego a inicios de los años 30 del siglo veinte, y el bosque secundario dominado por $N$. dombeyi (BSNd) fue originado luego de un incendio severo que ocurrió cerca de 1914 (González et al. 2015). Diversos atributos de composición y estructura de estos bosques son reportados en Ponce et al. (2017). Ponce (2014) generó un índice de bosque adulto, a través del cual se cuantifica cuán cercana es la composición y estructura de un determinado bosque con respecto de aquella de un bosque adulto. El BSMS tiene un valor de apenas 5\%, y el BSNd de $28 \%$. En función de que los tres bosques muestreados se encuentran en condiciones muy homogéneas de clima, suelo y topografía, y de las diferencias en el origen a los dos bosques secundarios (tipos de perturbaciones y edad), acá hipotetizamos que si bien los bosques sucesionalmente más avanzados (BA>BSNd>BSMS) pueden tener una mayor riqueza de especies vasculares debido a su mayor diversidad estructural (vertical y horizontal; e.g., Gutiérrez \& Huth 2012), los bosques secundarios pueden lograr niveles similares de riqueza ya sea por su estado de desarrollo o por su origen. Un bosque secundario en la fase de "Reinicio de sotobosque" (BSNd) permite una mayor llegada de 

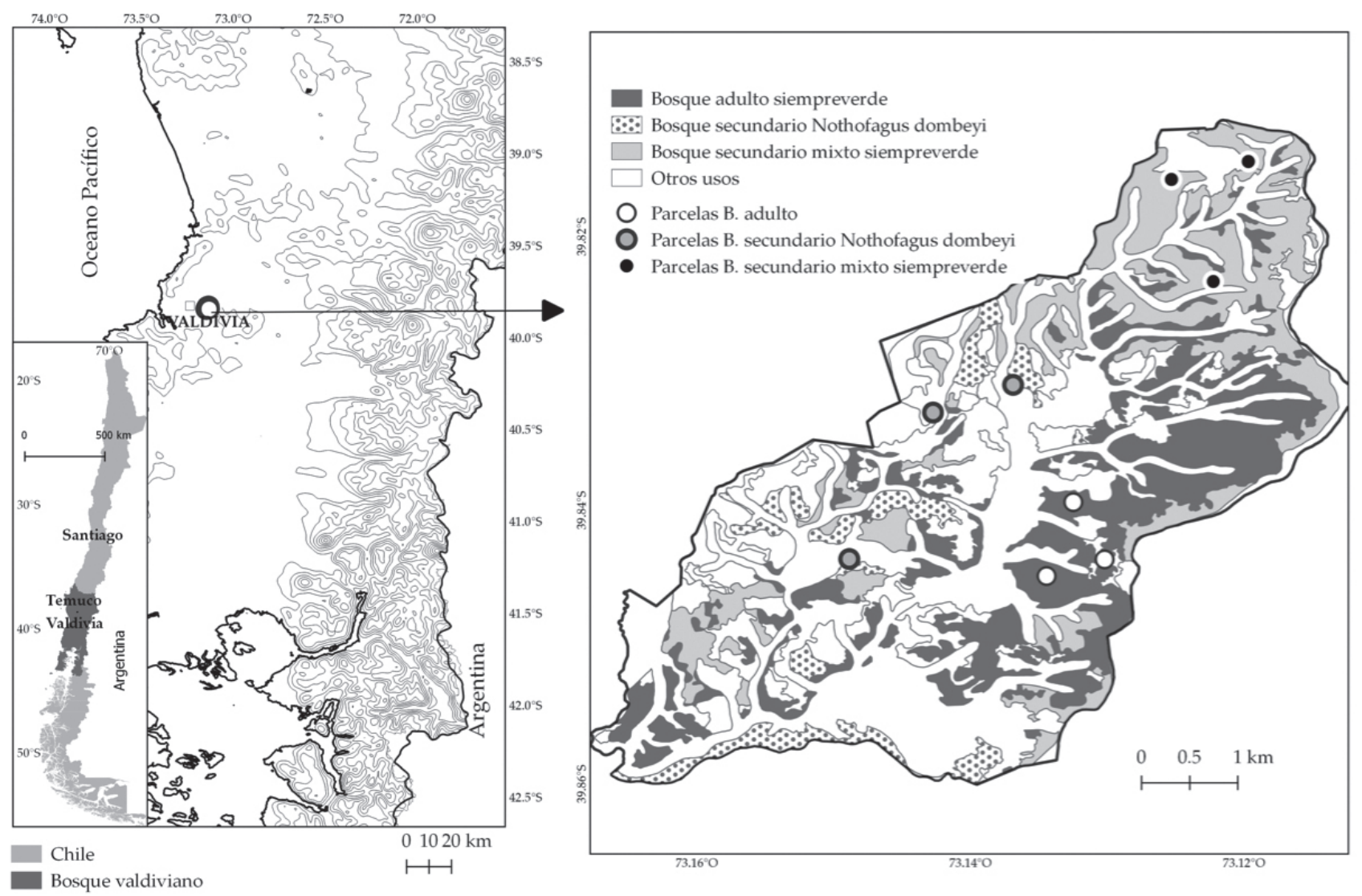

FiguRA 1. Ubicación geográfica, deslindes, y cobertura de bosques en el sitio experimental Llancahue, donde se muestra la ubicación de las nueve parcelas de 1 ha muestreadas en este estudio. / Geographical location, borders and tree cover in the Llancahue experimental site, showing the location of the nine 1-ha plots sampled in this study.

luz al suelo que un bosque secundario en una fase más temprana como la de "Exclusión fustal" (sensu Oliver \& Larson 1996), es decir se esperaría en el primer caso una mayor riqueza de especies en el sotobosque. Sin embargo, un bosque secundario que se origina por una interacción de perturbaciones (BSMS) que deja una mayor cantidad de legados biológicos, permitiría una mayor riqueza de especies durante el período de recolonización del ecosistema.

Dentro de cada unidad muestral se instalaron 100 subunidades circulares de $2 \mathrm{~m}^{2}$ para el registro de la regeneración de especies arbóreas y de cobertura de especies del sotobosque. Las subunidades fueron distribuidas sistemáticamente cada $10 \mathrm{~m}$ de distancia en cada eje de la parcela. En cada subunidad se registró la densidad de la regeneración de especies arbóreas, según categorías de altura $(5-<50 ; 50-<100 ; 100-<200$ y $>200)$ y la cobertura de especies no arbóreas del sotobosque.

Se determinó el promedio de la cobertura porcentual de cada especie, así como su frecuencia en las parcelas muestreadas, y con estas dos variables relativizadas el valor de importancia (Donoso 2015). El sotobosque de cada bosque fue comparado mediante la riqueza de especies y el Índice de similitud de Jaccard (Ecuación 1), este último para cada una de las tres comparaciones posibles (BA vs. BSNd, BA vs. BSMS y BSNd vs BSMS).

$$
\text { Indice de Jaccard }=\frac{\text { Especies comunes }}{(\text { Total de especies A+Total de especies B-especies comunes en A y B) }} * 100
$$


Cada uno de los bosques secundarios tiene 28 especies arbóreas y arborescentes, mientras que el bosque adulto tiene 18 especies arbóreas y arborescentes (Tabla 1). El BSMS registra una muy alta densidad de árboles $(>4.000$ por hectárea), un diámetro medio muy delgado, y está dominado por una mezcla de especies siempreverdes (Tabla 1). El BSNd está dominado por Nothofagus dombeyi y tiene una densidad más cercana al bosque adulto (entre 1.000 y 2.000 árboles por hectárea). Sin embargo, ambos bosques secundarios tienen un área basal similar, y muy inferior a la del bosque adulto. El BA tuvo la mayor riqueza de especies (29), seguido del BSNd (25) y del BSMS (20) (Tabla 2). El sotobosque estuvo dominado por muy pocas especies con valores de cobertura $>1 \%$ cuyo valor de importancia, derivado de ser especies muy frecuentes en cada rodal, fue $>$ $10 \%$ (Tabla 2). Tanto en el BA como en el BSNd la especie con mayor cobertura fue Nertera granadiensis, pero fue una especie de muy baja cobertura en el BSMS, donde la especie de mayor cobertura fue Boquila trifoliolata. Las especies de mayor cobertura (cerca o mayor a 1\%) comunes en los tres bosques fueron Chusquea quila, Luzuriaga sp. y Lapageria rosea. De acuerdo al Índice de Jaccard la similitud mayor ocurrió entre los dos bosques secundarios (BSNd y BSMS) con un $50 \%$, seguido del BSNd con el BA $(42,11 \%)$ y del BSMS con el BA $(36,11 \%)$. En comparación al estudio de Sink (2009), que también analizó los tres tipos de bosques evaluados en el presente estudio (pero con una intensidad de muestreo diferente) en Llancahue, los valores de riqueza de especies en general son muy similares, con cerca de 60 especies, y sólo para el BSMS el presente estudio reportó un valor menor de especies del sotobosque ( 20 vs. 32 reportadas en Sink (2009)).

La regeneración arbórea fue mayor en el BA, seguida del BSMS y del BSNd en todas las categorías de altura, y en todos los bosques fue disminuyendo desde las categorías de altura menores hacia las mayores (Fig. 2). Para la categoría de menor altura de la regeneración $(5-<50 \mathrm{~cm})$ tanto en BA como en BSMS dominaron especies tolerantes y semitolerantes, mientras que en el BSNd las tolerantes fueron las de menor densidad. Sin embargo, para las categorías de mayores alturas en todos los bosques fueron las especies intolerantes a la sombra las que tuvieron menor densidad. La categoría de mayor altura, la de brinzales o regeneración establecida, tuvo entre 5.000 y 6.000 individuos por hectárea para los BA y BSMS con un número ascendente desde las especies intolerantes hacia las tolerantes, pero el BSNd tuvo sólo cerca de 1.000 brinzales distribuidos en forma similar entre especies de distinta tolerancia. Sólo para las dos categorías de altura mayores hubo diferencias significativas, y en ambos casos el BSNd registró valores menores.

En resumen, los bosques de Llancahue albergan 30 especies arbóreas o arborescentes y 41 especies de arbustos, hierbas, enredaderas y helechos. Las diferencias y similitudes encontradas entre cada uno de los tres bosques estudiados ilustran los cambios en riqueza y composición de especies a través de la sucesión, incluyendo especies cuya fidelidad es mayor en ciertos estados sucesionales. Los dos bosques secundarios tienen más similitud entre ellos que con el bosque adulto y mayor riqueza de especies arbóreas y arborescentes. El bosque adulto tiene mayor riqueza de especies del sotobosque, y ésta es muy similar a los valores reportados por Donoso \& Nyland (2005) en bosques adultos Siempreverdes de la Depresión Intermedia y ladera oriental de la Cordillera de la Costa entre los 39 y $40^{\circ} \mathrm{S}$. En cuanto a riqueza de especies arbóreas, evaluadas dentro de un mismo sitio y a través de distintos estados sucesionales de bosques, los resultados del presente estudio, con 30 especies, son comparables al del estudio de Aravena et al. (2002) en el norte de la Isla de Chiloé, con sólo 15 especies. Es decir,
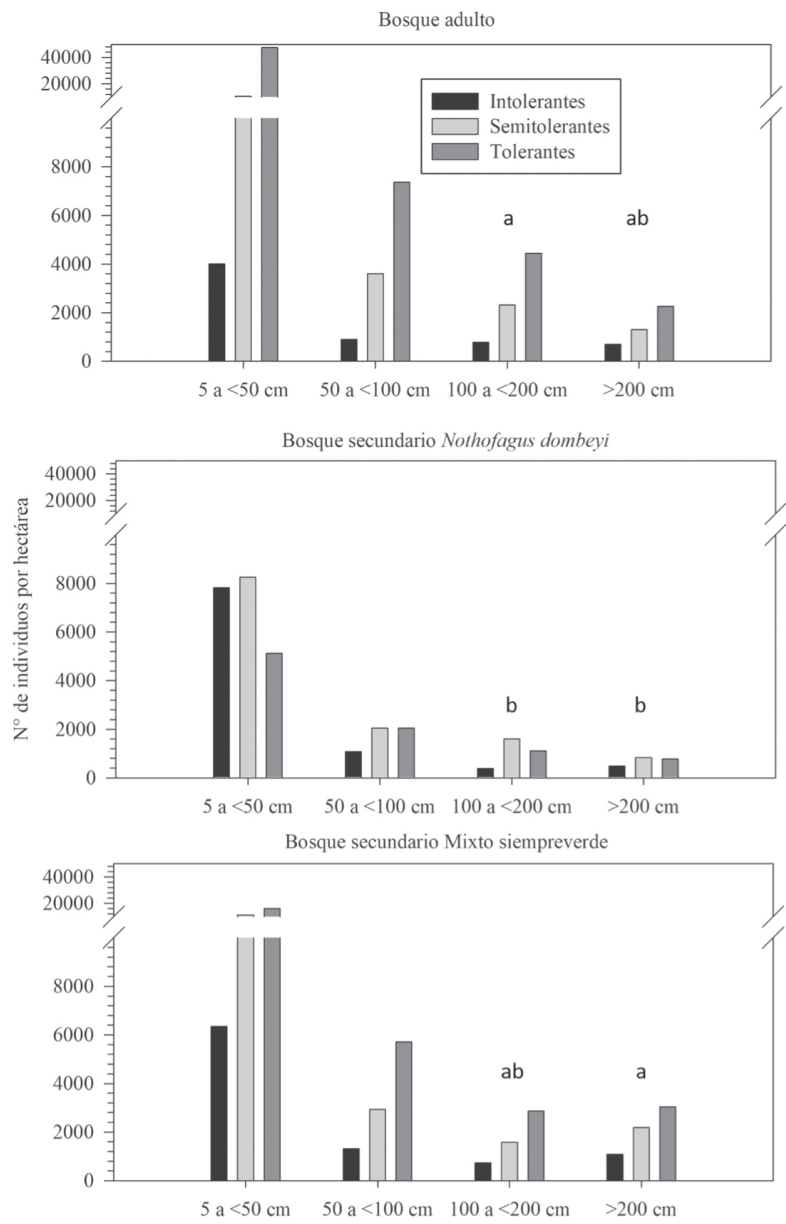

Figura 2. Densidad de plántulas y brinzales $(>200 \mathrm{~cm}$ de altura) de especies arbóreas y arborescentes en cada uno de los tres bosques muestreados y según tolerancia a la sombra en el sitio experimental Llancahue. Letras diferentes dentro de una categoría de altura reflejan diferencias significativas en densidad $(\mathrm{p}<0,05)$. / Seedling and sapling $(>200 \mathrm{~cm}$ in height) density of tree and arborescent species in each of the three forests studied and according to shade tolerances in the Llancahue experimental site. Different lettes within a same category indicate significant differences in density $(p<0,05)$. 
TABLA 1. Valor de importancia de cada especie arbórea o arborescente, densidad y área basal por hectárea (para individuos $\geq 5 \mathrm{~cm}$ en $\mathrm{DAP}$ ), y diámetro medio cuadrático (DMC, diámetro del árbol de área basal media) para cada uno de los tres bosques estudiados en el sitio experimental Llancahue./Importance value of the species, tree density and basal area per hectare (for individuals $\geq 5 \mathrm{~cm}$ in Diameter at Breast Height), and quadratic mean diameter (DMC, diameter of the tree with mean basal area) for each of the forests studied in the Llancahue experimental forest.

\begin{tabular}{|c|c|c|c|c|}
\hline ESPECIE & $\begin{array}{c}\text { TOLERANCIA A LA } \\
\text { SOMBRA* }\end{array}$ & $\mathrm{BA}$ & BSNd & BSMS \\
\hline Aextoxicon punctatum Ruiz \& Pav. & Tolerante & 23,60 & 1,43 & 1,36 \\
\hline Amomyrtus luma (Molina) D.Legrand \& Kausel & Tolerante & 0,61 & 5,31 & 7,04 \\
\hline Amomyrtus meli (Phil.) D. Legrand \& Kausel & Tolerante & 1,51 & 3,47 & 25,58 \\
\hline Aristotelia chilensis (Molina) Stuntz & Intolerante & - & 0,07 & 0,06 \\
\hline Caldcluvia paniculata Cav. (D.Don) & Tolerante & - & 0,31 & 0,02 \\
\hline Dasyphyllum diacanthoides (Less.) Cabrera & Semitolerante & 0,99 & 0,47 & 3,25 \\
\hline Drimys winteri J.R.Forst. \& G.Forst. & Semitolerante & 1,93 & 10,24 & 3,24 \\
\hline Embothrium coccineum J.R.Forst. \& G.Forst. & Intolerante & - & 0,02 & 2,56 \\
\hline Eucryphia cordifolia Cav. & Semitolerante & 16,78 & 25,30 & 18,42 \\
\hline Gaultheria mucronata (L.f.) Hook. \& Arn. & Intolerante & - & - & 0,01 \\
\hline Gevuina avellana Molina & Semitolerante & 1,45 & 3,71 & 9,47 \\
\hline Laurelia sempervirens (Ruiz \& Pav.) Tul. & Semitolerante & 0,25 & 0,75 & - \\
\hline Laureliopsis philippiana (Looser) Schodde & Tolerante & 43,92 & 2,55 & 5,90 \\
\hline Lomatia dentata (Ruiz \& Pav.) R.Br. & Tolerante & 0,05 & 0,58 & 0,55 \\
\hline Lomatia ferruginea (Cav.) R.Br. & Intolerante & 0,74 & 0,95 & 4,05 \\
\hline Lomatia hirsuta Diels ex J.F.Macbr. & Intolerante & - & 0,45 & 1,40 \\
\hline Luma apiculata (DC.) Burret & Tolerante & - & 0,02 & 0,34 \\
\hline Myrceugenia ovata (Phil.) L.E.Navas & Tolerante & 0,07 & 0,04 & 1,84 \\
\hline Myrceugenia parvifolia (DC.) Kausel & Semitolerante & - & 2,05 & - \\
\hline Myrceugenia planipes (Hook. \& Arn.) O.Berg & Tolerante & 2,74 & 0,16 & 2,01 \\
\hline Nothofagus dombeyi (Mirb.) Oerst. & Intolerante & - & 30,43 & 0,01 \\
\hline Ovidia pillopillo (Gay) Meisn. & Intolerante & - & 0,04 & 2,55 \\
\hline Persea lingue (Ruiz \& Pav.) Nees & Tolerante & 0,56 & 1,76 & 2,63 \\
\hline Podocarpus nubigenus Lindl. & Semitolerante & - & 1,48 & 0,06 \\
\hline Podocarpus salignus D.Don & Semitolerante & 0,35 & 3,20 & 2,17 \\
\hline Raukaua laetevirens (Gay) Frodin & Intolerante & - & 0,44 & 0,18 \\
\hline Rhaphithamnus spinosus (Juss.) Moldenke & Intolerante & 1,63 & 0,71 & 2,05 \\
\hline Saxegothaea conspicua Lindl. & Tolerante & 0,09 & 3,60 & 2,95 \\
\hline Tepualia stipularis (Hook. \& Arn.) Griseb. & Semitolerante & - & - & 0,01 \\
\hline Weinmannia trichosperma Cav. & Intolerante & 2,72 & 0,47 & 0,28 \\
\hline Densidad total ( $n^{\circ}$ árboles/ha) & & 1142 & 1511 & 4154 \\
\hline Área basal total $\left(\mathrm{m}^{2} / \mathrm{ha}\right)$ & & 91,8 & 61,9 & 60,4 \\
\hline Diámetro medio cuadrático $(\mathrm{cm})$ & & 32,3 & 23,3 & 13,6 \\
\hline
\end{tabular}

*Tolerancias a la sombra basadas en Donoso 2015, Gutiérrez \& Huth 2012, Lusk 1999. 
TABLa 2. Valor de importancia (en base a cobertura y frecuencia) de las especies no arbóreas registradas en cada uno de los bosques estudiados en el sitio experimental Llancahue/Importance value (based on cover and frequency) of non-tree species registered in each of the forests studied in the Llancahue experimental site.

\begin{tabular}{|c|c|c|c|c|c|c|}
\hline & BA & & BSNd & & BSMS & \\
\hline ESPECIE & $\mathrm{CP}(\%)$ & VI & $\mathrm{CP}(\%)$ & VI & $\mathrm{CP}(\%)$ & VI \\
\hline \multicolumn{7}{|l|}{ HIERBAS } \\
\hline Acaena ovalifolia Ruiz \& Pav. & 0,01 & 0,1 & - & - & - & - \\
\hline Centella asiatica L. Urb. & 0,01 & 0,11 & - & - & - & - \\
\hline Cerastium arvense $\mathrm{L}$. & 0,003 & 0,09 & - & - & - & - \\
\hline Digitalis purpurea L. & - & - & 0,04 & 0,29 & - & - \\
\hline Fascicularia bicolor Mez & 0,13 & 0,9 & 0,14 & 0,61 & 0,02 & 0,34 \\
\hline Greigia sphacelate (Ruiz \& Pav.) Regel & - & - & 0,27 & 1,01 & 0,32 & 3,77 \\
\hline Hydrocotyle sp. & 0,45 & 3,30 & 0,19 & 0,93 & - & - \\
\hline Lobelia tupa $\mathrm{L}$. & 0,01 & 0,12 & - & - & - & - \\
\hline Nertera granadensis (Mutis ex L.f.) Druce & 5,97 & 23,83 & 9,37 & 28,71 & 0,13 & 1,37 \\
\hline Osmorhiza chilensis Hook. \& Arn. & 0,12 & 1,05 & 0,16 & 1,16 & - & - \\
\hline Prunella vulgaris $\mathrm{L}$. & 0,08 & 0,37 & - & - & - & - \\
\hline Ranunculus repens $\mathrm{L}$. & 0,01 & 0,10 & - & - & - & - \\
\hline Solanum valdiviense Dunal & 0,01 & 0,18 & - & - & - & - \\
\hline Taraxacum officinale (L.) Weber ex F.H.Wigg. & 0,05 & 0,22 & - & - & - & - \\
\hline Uncinia sp. & 0,73 & 4,63 & 0,57 & 4,25 & 0,64 & 8,57 \\
\hline Viola reichei Skottsb. & 0,01 & 0,11 & - & - & - & - \\
\hline Viola rubella $\mathrm{Cav}$. & 0,02 & 0,21 & 0,06 & 0,64 & - & - \\
\hline \multicolumn{7}{|l|}{ Enredaderas } \\
\hline Asteranthera ovata (Cav.) Hanst. & - & - & 0,08 & 0,29 & - & - \\
\hline Boquila trifoliolata (DC.) Decne. & 0,47 & 3,45 & 2,99 & 15,18 & 1,06 & 16,03 \\
\hline Cissus striata Ruiz \& Pav. & 0,35 & 3,81 & 0,60 & 4,22 & 0,17 & 2,90 \\
\hline Cynanchum sp. & - & - & 0,03 & 0,37 & 0,01 & 0,48 \\
\hline Elytropus chilensis A.DC. & 0,05 & 0,38 & 0,05 & 0,40 & 0,04 & 0,77 \\
\hline Griselinia racemose (Phil.) Taub. & 0,01 & 0,11 & 0,13 & 0,68 & 0,02 & 0,34 \\
\hline Hydrangea serratifolia (Hook. et Arn.) F. Phil. & 0,10 & 0,75 & 0,05 & 0,21 & - & - \\
\hline Lapageria rosea Ruiz \& Pav. & 0,53 & 5,41 & 1,29 & 9,05 & 0,50 & 9,08 \\
\hline Luzuriaga polyphylla (Hook.) Mebr. & 2,27 & 12,01 & 2,29 & 10,72 & 0,95 & 13,66 \\
\hline Luzuriaga radicans Ruiz \& Pav. & 3,19 & 19,29 & 1,04 & 6,11 & 0,55 & 8,99 \\
\hline Mitraria coccinea Cav. & 0,83 & 4,45 & 0,40 & 2,48 & 0,07 & 2,02 \\
\hline Sarmienta repens Ruiz \& Pav. & 0,14 & 1,10 & - & - & - & - \\
\hline \multicolumn{7}{|l|}{ Arbustos } \\
\hline Chusquea quila Kunth. & 1,72 & 10,46 & 0,87 & 5,22 & 0,83 & 11,65 \\
\hline Gaultheria mucronate (L.f.) Hook. \& Arn. & - & - & - & - & 0,003 & 5,88 \\
\hline Rhamnus diffuses Clos & - & - & - & - & 0,003 & 7,06 \\
\hline Rubus ulmifolius Schott & - & - & - & - & 0,003 & 0,23 \\
\hline \multicolumn{7}{|l|}{ Helechos } \\
\hline Adiantum chilense Kaulf. & - & - & 0,02 & 0,14 & - & - \\
\hline Asplenium dareoides Cav. & 0,04 & 0,43 & - & - & - & - \\
\hline Blechnum chilense (Desv.) Hieron. & - & - & 0,01 & 0,12 & - & - \\
\hline Blechnum hastatum Kaulf. & 0,50 & 2,96 & 1,17 & 6,22 & 0,25 & 5,53 \\
\hline Blechnum magellanicum (Desv.) Mett. & - & - & - & - & 0,10 & 1,08 \\
\hline Lophosoria quadripinnata (J.F.Gmel.) C.Chr. & - & - & 0,15 & 0,64 & 0,01 & 0,25 \\
\hline Megalastrum spectabile (Kaulf.) A.R.Sm. \& R.C.Moran & - & - & 0,02 & 0,35 & - & - \\
\hline Polypodium feuillei Bertero & 0,003 & 0,09 & - & - & - & - \\
\hline
\end{tabular}


en tres grados latitudinales hay un 50\% menos de especies arbóreas. La similitud en el número total de especies (entre 53 y 47) valida nuestra hipótesis en cuanto a que la riqueza de especies en un bosque no es sólo función de una mayor diversidad estructural como la que ocurre en bosques adultos (en este caso BA), sino que también de diferentes estados de desarrollo en bosques sucesionales, así como del tipo de perturbaciones que han dado origen a los bosques secundarios. Es decir, la mezcla y data de perturbaciones naturales (por ejemplo formación de claros por muerte de árboles por competencia) y antrópicas (fuego, tala), además del estado sucesional, son determinantes sobre la riqueza de especies en estos bosques costeros. Sería de alto valor ecológico y social poder ampliar este tipo de comparaciones en el paisaje del centro-sur de Chile.

\section{AGRADECIMIENTOS}

Al proyecto FONDECYT N 1150496.

\section{REFERENCIAS}

Aravena, J.C., Carmona, M.R., Pérez, C.A., Armesto, J.J. 2002. Changes in tree species richness, stand structure and soil properties in a successional chronosequence in northern Chiloé Island, Chile. Revista Chilena de Historia Natural 75: 339-360.

CIREN. 1999. Estudio agrológico de la Provincia de Valdivia - X Región; Santiago, Chile. 199 pp.

Donoso, C. 2015. Estructura y Dinámica de los Bosques del Cono Sur de América. Universidad Mayor, 405 pp.

Donoso, P.J., Nyland, R.D. 2005. Seeding density according to structure, dominance and understory cover in old-growth forest stands of the evergreen forest type in the coastal range of Chile. Revista Chilena de Historia Natural 78(1): 51-63.
Donoso, P.J., Frene. C., Flores, M., Oyarzún, C., Moorman, M., ZAVALETA, J. 2014. Balancing water supply and old-growth forest conservation in the lowlands of south-central Chile through an adaptive co-management approach. Landscape Ecology 29(2): 245-260.

González, M.E., Szejner, P., Donoso, P.J., Salas-Eljatib, C. 2015. Fire, logging and establishment patterns of secondgrowth forests in south-central Chile: implications for their management and restoration. Ciencia e Investigación Agraria 42(3): 427-441.

Gutiérrez, A.G., Huth, A. 2012. Successional stages of primary temperate rainforests of Chiloé Island, Chile. Perspectives in Plant Ecology, Evolution and Systematics 14(4): 243256.

Lara, A., Zamorano, C., Miranda, A., González, M., Reyes, R. 2016. Bosque Nativo. En: Informe País. Estado del Medio Ambiente en Chile, pp. 167-210. Centro de Políticas Públicas, Universidad de Chile, Santiago, Chile.

Locher-Krause, C., Volk, M., Walker, B., Thonfeld, F. 2017. Expanding temporal resolution in landscape transformations: Insights from a landsat-based case study in Southern Chile. Ecological Indicators 75: 132-144.

Luebert, F., Pliscoff, P. 2006. Sinopsis bioclimática y vegetacional de Chile. Editorial Universitaria, Santiago, Chile. 316 pp.

Oliver, C., Larson, B. 1996. Forest Stand Dynamics. John Wiley and Sons, Inc., New York, USA. 520 pp.

Ponce, D.B. 2014. Comparación de atributos estructurales y de composición entre bosques adultos y bosques secundarios en la Depresión Intermedia del centro-sur de Chile. Tesis Magíster en Ciencias, Mención Recursos Forestales, Universidad Austral de Chile, Valdivia, Chile. 62 pp.

Ponce, D.B., Donoso, P.J., Salas-Eluatib, C. 2017. Differentiating Structural and Compositional Attributes across Successional Stages in Chilean Temperate Rainforests. Forests 8(9): 329.

SINK, S. 2009. Managing for Old-Growth Conditions in SecondGrowth Temperate Rainforests of South-Central Chile. Ph.D. Dissertation, College of Forestry and Natural Resources, North Carolina State University, Raleigh, North Carolina, USA. 68 pp.

Recibido: 05.04.2018

Aceptado: 22.08.2018 